$$
\text { Paper }
$$

\title{
Astrometric calibration of the Clementine meridian line (1702) of S. Maria degli Angeli on the zodiacal signs, in the IGEA observational campaign (2018/21)
}

\author{
Costantino Sigismondi ${ }^{1,2,3}$ \\ Published: date \\ Academic Editor: name \\ 1 ICRA/Sapienza Univeristy of Rome; \\ 2 Ateneo Pontificio Regina Apostolorum, Rome; \\ ${ }^{3}$ ITIS Galileo Ferraris, Rome \\ * Correspondence: sigismondi@icra.it
}

Abstract: The meridian line is a basic instrument for positional astronomy, it was used to study the motion of Sun, Moon, planets and the position of stars by measuring position and time of their passage through the meridian plane. The accuracy of such positions was dependent on precise theories of the atmospheric refraction (Cassini, 1655 and Laplace, 1825) and by the use of reference marks present originally on the meridian line, and now cancelled by the centuries. From October 27, 2018 the new pinhole of the meridian line in the Basilica of S. Maria degli Angeli in Rome (1702) is a circle $25 \mathrm{~mm}$ wide and $6.11 \mathrm{~mm}$ thick and its position is fixed, in order to perform a series of observations of astrometric quality, the IGEA campaign. The comparison of the observed positions of the meridian passages of the Sun, Southern and Northern limbs, with the ephemerides of Calsky.org and Stellarium 0.20.2 for the Sun are examined for the dates of the ingresses into the zodiacal signs, when the ecliptic longitude is exactly $0^{\circ} / 180^{\circ}$ (Aries and Libra, spring and fall equinox), 30\% $150^{\circ}$ (Taurus, Virgo), 60\% $120^{\circ}$ (Gemini, Leo), $90^{\circ}$ (Cancer), 330 $/ 210^{\circ}$ (Pisces, Scorpio), $300^{\circ} / 240^{\circ}$ (Aquarius/Sagittarius), $270^{\circ}$ (Capricorn). The former geometrical calibration of the marks present on the line, with a total station, is compared with another calibration done with a metal and laser meter. The first star on the floor of the Basilica representing the position of the Sun on August 20, 1702 when the pope Clement XI visited the meridian line, financed by him, has been calibrated with the solar image. The present pinhole is $4.4 \pm 0.1 \mathrm{~mm}$ South with respect to the original one of 1702 .

Keywords: ephemerides; refraction; solstices; equinoxes; equation of time; Earth's rotation; obliquity; eccentricity; penumbra; zodiacal sign; gnomonics; ecliptic longitude; declination; meridian transit.

\section{Introduction: The Clementine Gnomon and the solar astrometry in $\mathbf{1 7 0 2}$}

The paper on Reference system effects detected with meridian astronomy on the Clementine Gnomon (Rome, 1702), published in this series in February 2021, contains already a brief introduction to this Great 
Gnomon, as it was called by the astronomer Francesco Bianchini (1662-1729) who completed it in 1702. The Clementine Gnomon is located in the Basilica of S. Maria degli Angeli e dei Martiri in Rome, built by Michelangelo in 1561-1564 in the Diocletian Baths and exploiting the still existing hall $30 \mathrm{~m}$ tall. The $25 \mathrm{~mm}$ circular pinhole, which is the objective of this lensless telescope, is at height of $20.3534 \mathrm{~m}$, the line up to the Capricorn is $44.917 \mathrm{~m}$ long; the accuracy of the measured positions of the solar limbs on the meridian line is $\pm 1 \mathrm{~mm}$, including the turbulence effects of the atmosphere, mainly occurring near the pinhole itself, for the strong heating of the Sun over the external bricks, which makes the air boiling. In order to achieve the accuracy to detect relativistic effects on the motion of the Earth around the Sun, a precise calibration of this unique instrument is being performed. This is the subject of this second paper. The accuracy of a second of arc, or a few arcsecs, is our goal. The status of this calibration is here discussed, presenting the situation of this historical instrument, the only one of his epoch still in use with astrometric measurements. The other meridian lines in Italy are in S. Petronio (Bologna, Cassini 1655), in the Cathedral of Palermo (Piazzi, 1801) and in the Dome of Milan (De Cesaris, 1782). The one of Le Monnier in Paris SaintSuplice (1642) completes the scenario (for all see Heilbron, 1999). A main restauration of the meridian line has been carried in 1999-2000 for the Jubilee year, without a correction in the pinhole, which had been modified arbitrarily in the previous years (Catamo and Lucarini, 2012). In the following text, the new circular pinhole of the IGEA observational campaign (Informatized Geometric Ephemerides for Astrometry) is described. In the past (2002-2018) smaller circular pinholes were adapted to the existing, and irregular one, for special public observations. The present circular shape and its dimension allow astrometric measurements, with enough light also in winter, when the solar spots have been visibile on the lensless solar image projected on the floor's marbles.

\section{The new IGEA campaing (2018-on) and the pinhole's seasonal shadowing}

The position of the pinhole is determining the position of the observed solar image on the floor of the Basilica. The center of the image is connected to the center of the pinhole by a line inclined exactly as the altitude of the center of the Sun, as refracted by the atmosphere.

The effective center of the pinhole is always $1.25 \mathrm{~mm}$ shifted toward South, because of the Northern indent of fig. 2, and is moved 3.11· $\tan (\mathrm{z}) \mathrm{mm}$ Northward by the shadowing of the pinholes' thickness. The zenithal angle $z=90^{\circ}-h$, where $h$ is the altitude of the Sun at the meridian.

The IGEA acronym stands for Informatized Geometric Ephemerides for Astrometry and it is remembering the dedication to Igea Contessa (27.X.1918-9.12.2008) in her centennial, 27.X.2018, starting date of this astrometric campaign. 


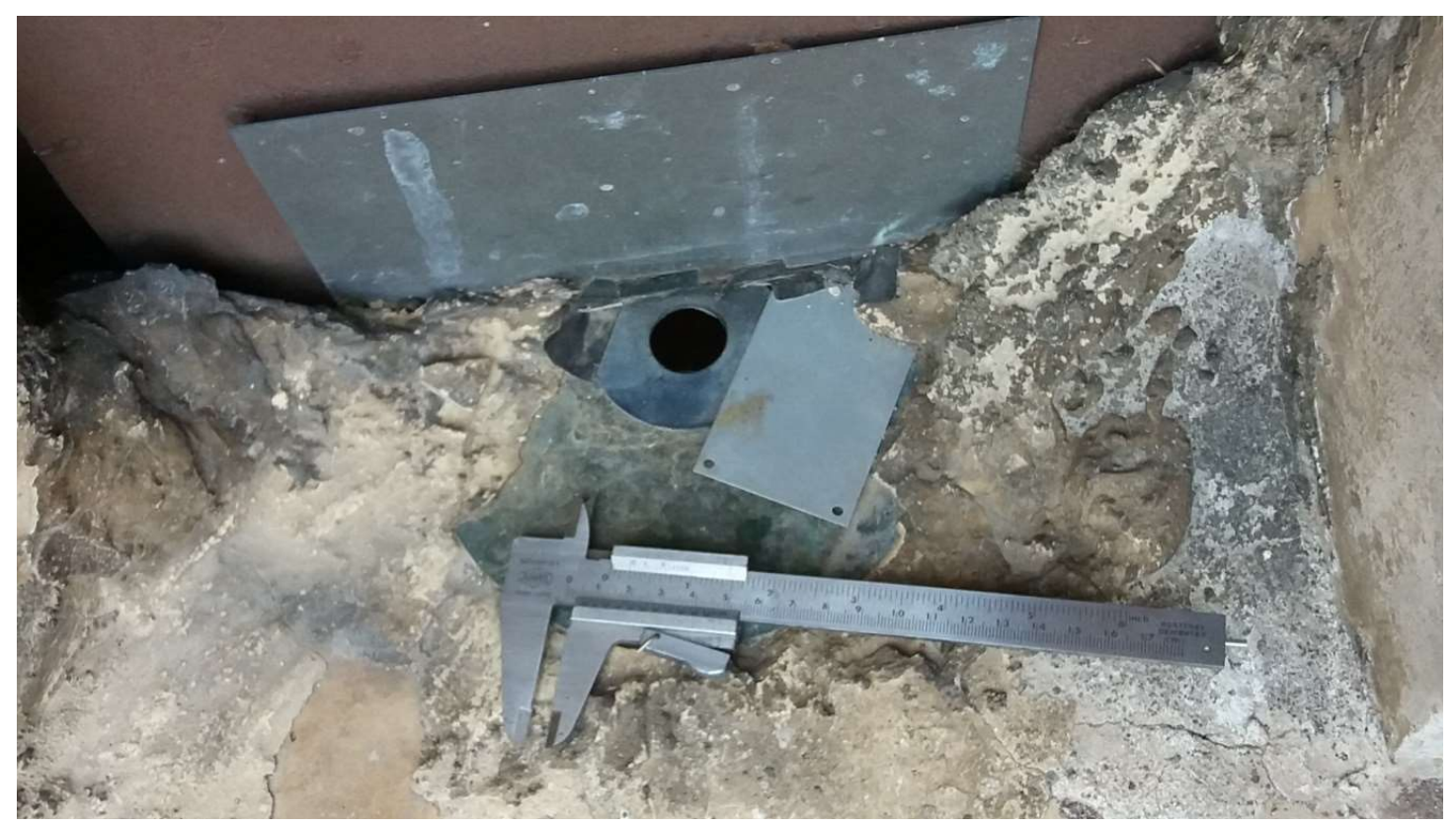

Figure 1. The pinhole of the meridian line, as seen from the outside of the Basilica. The North is here up right. This external area of brick and cement determines the air turbulence of the solar image.

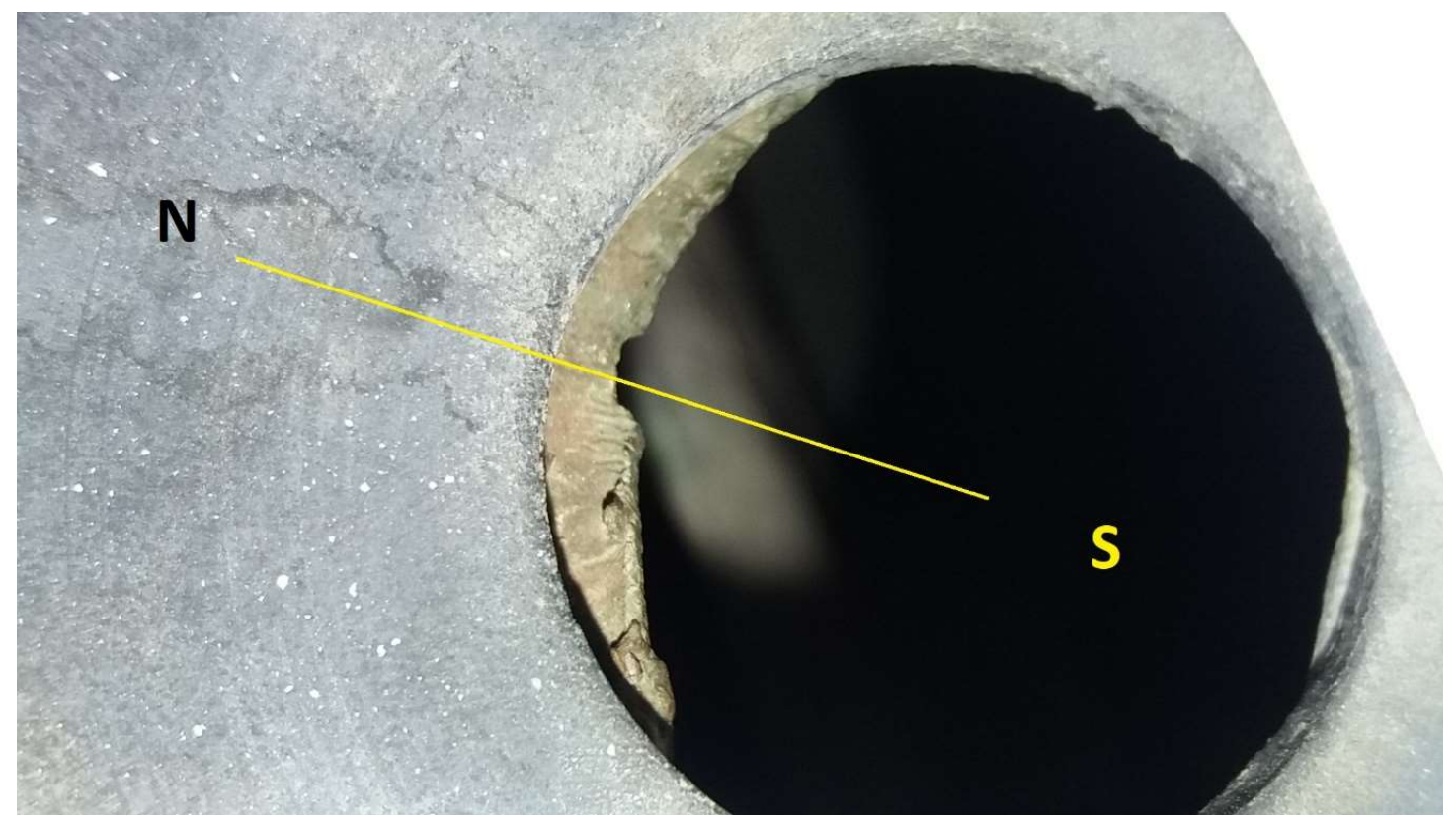

Figure 2. The pinhole of the meridian line, as seen from the outside of the Basilica. In this image, magnified with respect to fig. 1, the North is left and a $2.5 \mathrm{~mm}$ indent made by the original underlying bronze pinhole (found deformed and so re-established in its circular shape). The stainless steel pinhole is $25 \mathrm{~mm}$ wide and $6.11 \mathrm{~mm}$ thick, including the underlying original bronze. The pinhole area has been painted in white to reduce the solar heating and the local air turbulence, without obtaining a significant improvement: the turbulence is greatly amplified by this optics.

\section{The observed ingresses of the Sun in the zodiacal signs and the four anni cardines}


The great meridian line is traditionally related to the anni cardines, the cornerstones of the tropical year, i.e. the winter solstice, the spring equinox, the summer solstice and the fall equinox.

Many people come to the Basilica in these dates to see the meridian transit of the Sun to witness the beginning of the new season. These seasons correspond to the ingress to the zodiacal signs of Capricorn, Aries, Cancer and Libra, according to the traditional division of the ecliptic into 12 equal parts.

Their names are related to the Equinox of the Epoch, and their declinations change slightly every year because the obliquity of the ecliptic is changing, form $23^{\circ} 28^{\prime} 40^{\prime \prime}$ in 1702 to $23^{\circ} 26^{\prime} 09^{\prime \prime}$ in 2021, with a $\pm 9^{\prime \prime}$ oscillation of 18 years' period due to the lunisolar nutation.

The precession of the equinoxes is also acting, but the Gregorian reformation of the calendar reduces its effect on the equinoxes' dates almost completely on a 400 years base, only the 4 year oscillation remains on the ingress' dates. The declination of the signs depend on their ecliptic longitude and on the obliquity of the ecliptic.

In principle, when the passages of the Sun are compared with a precise ephemerides, each daily observation at the meridian is equivalent to another, but the 12 ingresses into the zodiacal signs includes the 4 anni cardines and have also a public impact.

The two equinoxes, two of the anni cardines, are independent on the obliquity, but only from the latitude of the pinhole, which does not change in few hundred years. Their measurement depends on the accuracy of the determination of the latitude, and Bianchini included on it the stellar aberration suffered by the Polaris in the first week of January. He measured $41^{\circ} 54^{\prime} 27^{\prime \prime}$ the apparent altitude of the Celestial North Pole as the center of Polaris's daily orbits, while the real latitude is $41^{\circ} 54^{\prime} 11^{\prime \prime}$. The $16^{\prime \prime}$ difference is due to the stellar aberration, a special relativistic effect whose consequences are detectable with this meridian line. A higher latitude means lower meridian culmination of the celestial equator, and consequently the position of the equator on the floor of the Basilica is shifted by $2.9 \mathrm{~mm}$ toward the base of the pinhole, i.e. Southward.

This shift implies a delay of the estimated equinox in spring, when the Sun's image moves from the Southern hemisphere to the North, and reversely on the floor because of the pinhole's projection mechanism. In the fall equinox, conversely, the equinox is anticipated for the opposite reason.

This geometrical situation occurs also for the other zodiacal signs.

The timing of the two solstices, the other two cardines, are obtained by fitting a quadratic function in the time and position of the limbs, to find the turning point, corresponding to the minimum of the parabola. Their positions depend strongly on the obliquity of the date.

The zodiacal signs are corresponding to $30^{\circ}$ sectors of the ecliptic longitude: respectively $0^{\circ} / 180^{\circ}$ (Aries, spring equinox and Libra, fall equinox), 30\% $/ 150^{\circ}$ (Taurus, Virgo), 60\% $120^{\circ}$ (Gemini, Leo), $90^{\circ}$ (Cancer), 330\% $/ 210^{\circ}$ (Pisces, Scorpio), 300\% $/ 240^{\circ}$ (Aquarius/Sagittarius), $270^{\circ}$ (Capricorn), and, excepted the solstices, all are computable by linear interpolation and assuming, in first approximation, a constant angular velocity of the Sun in declination between the interpolating observations. 


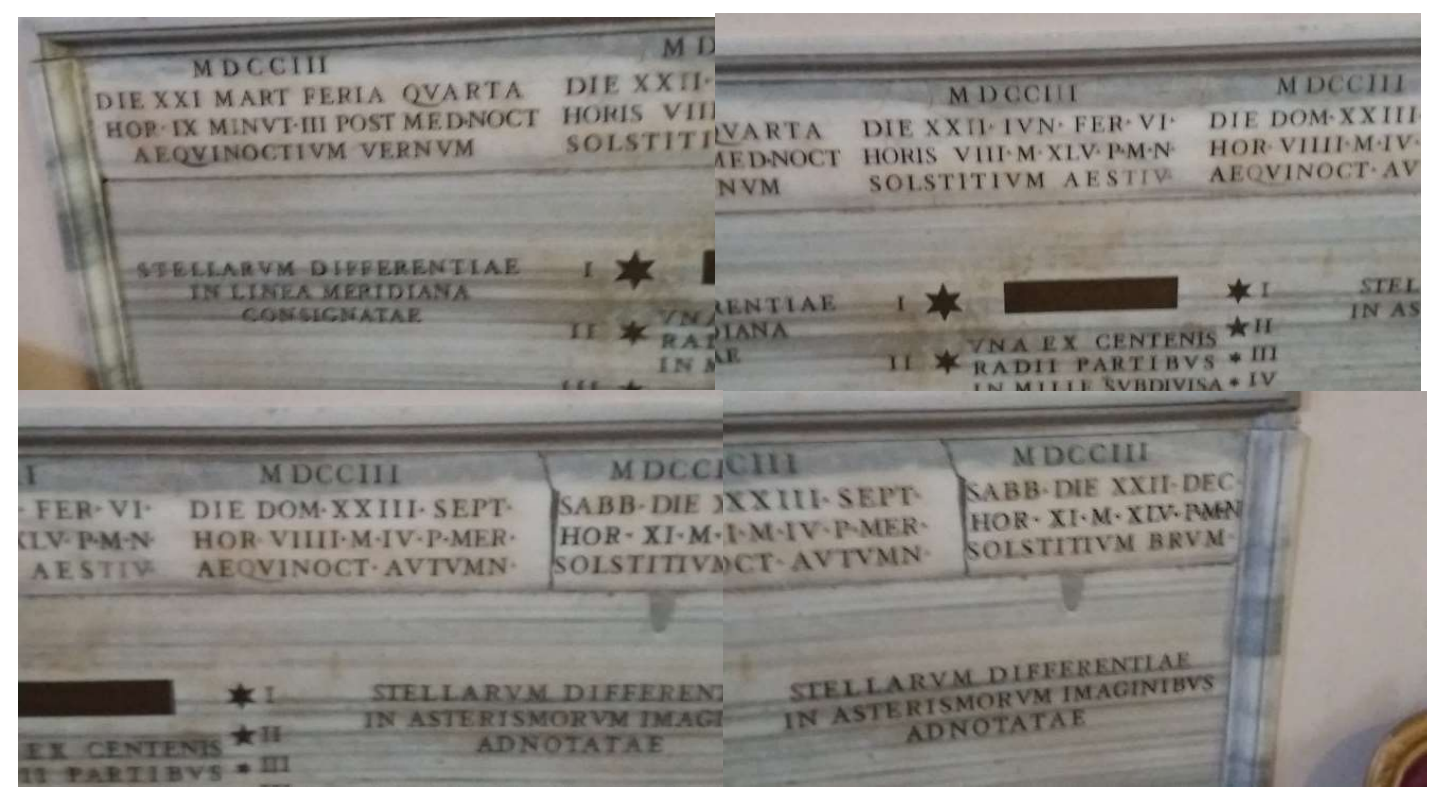

Figure 3-6. The inscription in the presbyterium of the Basilica, with the Anni Cardines measured in 1703. Vernum is spring, Aestivum is summer, Autumnalis is fall, Brum. stands for Brumalis and it is winter. The timing is given up to the nearest minute and the P.M. is post meridiem, i.e. after the solar meridian transit, while post mediam noctem is after the opposite transit, half true solar day after.

Francesco Bianchini published these instants to the nearest minute of time, on the marble and on a book The Nummo et Gnomone Clementino (Roma, 1703) where he declared an accuracy of 10 minutes on these timing and of 1/1000 of a centesimal part in the recovery of both solar limbs on the meridian line. That is meaning $0.2 \mathrm{~mm}$ of declared accuracy for such naked eye observations. In his book Bianchini described in depth some observations near the solstices and the one of $6^{\text {th }}$ October 1702 in the occasion of the inauguration of the meridian line by the pope Clement XI, who was celebrating in the same day the second anniversary of his first solemn mass celebrated there after his presbyteral ordination.

The possibility to assess the parameters of the Gregorian reformation of the calendar (1582) after only one year of observations with this meridian line, financed by the Cardinal Gianfrancesco Albani, elected pope on 23 November 1700 with the name of Clement XI, was possibile by calculating the average tropical year between 1500 (Bernhard Walther 1430-1504 in Nuremberg) and 1703 , and obtaining an error of \pm 10 minutes divided by 203 tropical years, i.e. \pm 3 seconds.

The absolute accuracy of this Great Gnomon realized with the utmost technology of the epoch, 1700, has been tested in the present analysis with the new IGEA pinhole.

\section{The quality of the new IGEA observations}

All observations are documented with a continous video, synchronized with the UTC by filming a watch CASIO G-SHOCK radio controlled, with the signal received at 0 AM of the same day of the meridian transit. This is for verifying also the de-rotation of the Earth due to the progressive continental isostasy. For this study is also crucial that along the years, during which the $\Delta$ UT1 may change of 0.7 seconds, the pinhole remain fixed, because in that interval of time the motion of the image on the floor can be larger than $1 \mathrm{~mm}$. The accuracy in time is obtained by multiple averages of independent timings on lines parallel and very close to the meridian one (a few $\mathrm{cm})$, and it is $\pm 0.3 \mathrm{~s}$. 
Each complete observation consist in the times of contact of the image with the meridian line, and positions of the solar limbs on the meridian line.

Since the measurement with a total station cannot be performed each time, some marks have been calibrated and temporarily attached on the line, in order to be used daily. The position with respect these marks is reported at each observation, with an accuracy of $\pm 1 \mathrm{~mm}$. A linear fit of some positions of the limbs before and after the meridian transits is done visually with a rod, and the best fit, the average position, is reported on the line, reducing the nominal accuracy below $1 \mathrm{~mm}$. From winter to summer this corresponds to 2 " to $10^{\prime \prime}$ in the meridian positions, because the image's axis ranges from $1100 \mathrm{~mm}$ to $210 \mathrm{~mm}$ while the solar diameter ranges from 1952" to $1888^{\prime \prime}$.

In this paper the positional data of the limbs on the meridian line are transformed into angular altitude of the observed center of the Sun, and compared with the ones simulated by Stellarium 0.20.2 with and without atmosphere. In the first part of this campaign the calsky.org service was used, but this remarkable website is unfortunately no longer in function, and the substitution with Stellarium has been applied with very good results, consistent with calsky standards.

The simulation without atmosphere are compared with the observations to which the Cassini refraction $\mathrm{h}^{\prime}=\mathrm{h}-60^{\prime \prime} \cdot \tan \left(90^{\circ}-\mathrm{h}\right)$ is subtracted, or the Laplace one, which, beyond Cassini term, includes also the $0.067^{\prime \prime} \cdot \tan ^{3}\left(90^{\circ}-\mathrm{h}\right)$ cubic term in zenital distance $\mathrm{z}=90^{\circ}-\mathrm{h}$. In the previous paper on the relativistic effects detectable at this meridian line (ECU 2021, February 2021) the distances of the solar limbs from the end of the meridian line were published, for the winter solstice 2020 in Capricorn, and for Scorpius 2020, Sagittarius 2020 and Aquarius 2021. Here we proceed with the analysis of the altitudes of the center of the Sun for these zodiacal signs, extending to Pisces and Taurus 2021, and Gemini 2019 and Cancer 2020, to cover all the extension of the Meridian Line and using all the reference marks measured with the total station.

\section{The reference marks used in the IGEA campaign, from Cancer to Capricorn}

The marks on the meridian line have been placed and measured by G. Cultrera and P. Spera at the beginning of IGEA campagn in November 2018 with the students of G. Ferraris of Rome, CAT division, using a total station. Moreover a steel meter and a laser distantiometer calibration has been realized by me to understand some differences noticed by comparing the Ephemerides, the observations and the marks' positions.

The following table has been our reference table for this campaign.

The centesimal part of the 1702 pinhole's height is the reference of the measurements made along the meridian line. 
Table 1. The positions of the marks on the Clementine Gnomon used for the IGEA campaign.

\begin{tabular}{cccc}
\hline $\begin{array}{c}\text { Centesimal } \\
\text { Part (nominal } \\
\text { 1702) }\end{array}$ & $\begin{array}{c}\text { Distance from the } \\
\text { zero of 1702 [mm] }\end{array}$ & $\begin{array}{c}\text { Centesimal } \\
\text { Part (nominal } \\
\text { 1702) }\end{array}$ & $\begin{array}{c}\text { Distance from the } \\
\text { zero of 1702 [mm] }\end{array}$ \\
\hline 0 & 0,0 & 130 & 26441,5 \\
37 & 7534,5 & 135 & 27453,7 \\
40 & 8128,5 & 140 & 28486,9 \\
45 & 9156,2 & 145 & 29516,7 \\
50 & 10148,5 & 150 & 30525,7 \\
55 & 11167,2 & 155 & 31536,4 \\
60 & 12190,5 & 160 & 32559,2 \\
65 & 13207,2 & 165 & 33574,2 \\
70 & 14223,5 & 170 & 34594,6 \\
75 & 15239,8 & 175 & 35614,4 \\
80 & 16248,8 & 180 & 36628,7 \\
85 & 17266,0 & 185 & 37646,6 \\
Equator & 18250,3 & 190 & 38663,6 \\
90 & 18278,7 & 195 & 39681,1 \\
95 & 19290,9 & 200 & 40701,1 \\
100 & 20328,6 & 205 & 41724,4 \\
105 & 21344,8 & 210 & 42743,2 \\
110 & 22361,8 & 215 & 43759,7 \\
115 & 23388,8 & 216 & 43964,7 \\
120 & 24403,5 & 220 & 44777,6 \\
125 & 25418,7 & End of the Line & 44916,9 \\
\hline
\end{tabular}

5. The anni cardines extended to all zodiacal signs, and measures error's discussion

Table 3. The marks involved in the specific measurement during the ingresses to the zodiacal signs.

\begin{tabular}{|c|c|c|c|c|}
\hline Zodiacal Sign & $\begin{array}{l}\text { Observed ingress } \\
\text { local time Rome }\end{array}$ & $\begin{array}{c}\text { Ephemerid } \\
\text { es }\end{array}$ & $\begin{array}{c}\text { Reference } \\
\text { mark involved }\end{array}$ & $\begin{array}{l}\text { Pinhole's shift [mm] }{ }^{1} \\
\text { with respect to } 1702\end{array}$ \\
\hline SCORPIO & 23 Oct 2020 00:55 & $00: 59$ & 130 & +1.0 \\
\hline SAGITTARIUS & 21 Nov 2020 21:40 & 21:41 & 190 & -0.2 \\
\hline AQUARIUS & 18 Jan 2021 21:59 & 21:40 & $190^{2}$ & +4.4 \\
\hline PISCES & 19 Feb 2021 12:01 & 11:43 & $130^{2}$ & +4.4 \\
\hline ARIES & 20 Mar 2021 10:42 & 10:37 & EQUATOR & +0.4 \\
\hline ARIES/LIBRA & Mar-Sep 2019 & & EQUATOR & -4.3 \\
\hline TAURUS & 19 Apr 2021 22:30 & $22: 32$ & 60 & -0.4 \\
\hline GEMINI & 22 May 2019 07:58 & 09:57 & 40 & -6.1 \\
\hline CAPRICORN & 21 Dec 2020 10:37 & 11:02 & Parabolic fit ${ }^{2}$ & +4.3 \\
\hline CANCER & 21 Jun 2019 18:06 & $17: 54$ & Parabolic fit & -3.7 \\
\hline
\end{tabular}

${ }^{1}$ a positive shift is here intended toward South ${ }^{2}$ Measures from the end of the meridian line with steel meter

The times of the ingress in the various zodiacal signs can be considered as extension of the anni cardines. The shift of the pinhole along the meridian direction, assumed as positive Southward, is 
used as a free parameter to account for the delay or anticipation of the ingress' time observed with respect to the ephemerides. The various signs have been measured with interpolation between two passages nearby: Aquarius and Pisces were one day bracketing, e.g. 18 and 19 for AQR and 19-20 for PSC. Similarly for SCO and SGR, while 18 and 20 for TAU and 22 and 25 for GEM. The two solstices have been fitted with a parabola over several measurements, symmetrical to the solstice. The instant of the minimum, averaged from Southern and Northern limbs between 7 December and 4 January, is 21 December 2020 at 10:37 $\pm 4 \mathrm{~min}$. The time of the solstice in IMCCE ephemerides is at 11:02 TMEC, 25 minutes later. The causes of this difference are not dependent on the calibration of the meridian line, since the position of the limbs can be measured from each of the references on the line: the "tropic", i.e. the inversion of the motion of the Sun in declination, is universal. The corresponding pinhole's shift for winter and summer solstices is obtained by moving Southward the pinhole in order to have the Sun's height equal to the real one (the ephemerides' one) in both seasons. The values of the solstices are the result of a parabolic fit with 16 datapoints each one, while the ingresses into other zodiacal signs are the interpolation of two datapoints each one. The errorbars for the solstices are statistically $\sqrt{ } 16=4$ times smaller than the ones for each sign, which are the combination in quadrature of each daily errorbar.

This daily errorbar is typically within $\pm 10^{\prime \prime}$, two of them in quadrature are, in the worst cases $\pm 15^{\prime \prime}$, for each zodiacal sign, as a result of inter/extrapolation, and the solstices should be within $\pm 4^{\prime \prime}$ accurate.

From the table each zodiacal sign has a typical errorbar within $\pm 2.4 \mathrm{~mm}$.

Each daily measurement, has an accuracy which is below this value, because I tried always to measure the position of each limb within $\pm 1 \mathrm{~mm}$, and the center's accuracy is $\pm 1.4 \mathrm{~mm}$ as the result of the quadrature. An interpolation between two daily measurements includes the errors in quadrature of each day, then $\pm 2.0 \mathrm{~mm}$, consistent with the previous affirmation of $\pm 2.4 \mathrm{~mm}$ for each zodiacal sign ingress.

In the table 3 the advance/delay with respect to the ephemerides has been transformed into pinhole's shift, through the angular velocity in declination of the Sun and its projection to the floor in $\mathrm{mm} / \mathrm{min}$. Only AQR and PSC measures from the end of the line are in agreement with CAP parabolic fit, while the others differ because of statistical fluctuations, or very noisy data like GEM obtained with an extrapolation over 3 days with the second one affected by clouds. All data of zodiacal signs together are not enough accurate to determine the present pinhole position with respect to the one of 1702 unambiguosly and with a $0.1 \mathrm{~mm}$ accuracy. This is possible with a selection.

\subsection{Data selection critheria}

The shadowing effect of the cylindrical pinhole, is very well represented and found in all data, and the agreement with the ephemerides is complete for the data of Capricorn, Aquarius and Pisces, all recovered from the end of the line with a steel meter. The average of the measurements obtained with twin signs would be the logic way to proceed with this analysis, by taking account of the motion of the Sun from or toward the Northern hemisphere, as previously said for the equinoxes (section 3). A series of indices on systematic errors in the reference marks, ranging up to 45" as in the case of the last measurements made after Taurus' ingress up to 26 April 2021 (6 mm), with respect to the reference mark 55 used in three meridian transits, has suggested me to select the data. This notwithstanding a perfect result of the Taurus' ingress with a two minutes difference with the ephemerides $(0.4 \mathrm{~mm})$, which tells us that the systematic error for reference mark 60 is negligible, but it is not true for all reference marks in table 1.

Then from table 3, I consider useful to set the pinhole location only CAP AQR and PSC data, and the present pinhole is $4.4 \pm 0.1 \mathrm{~mm}$ shifted Southward with respect to 1702 .

\section{Past and present on the floor of the Basilica}




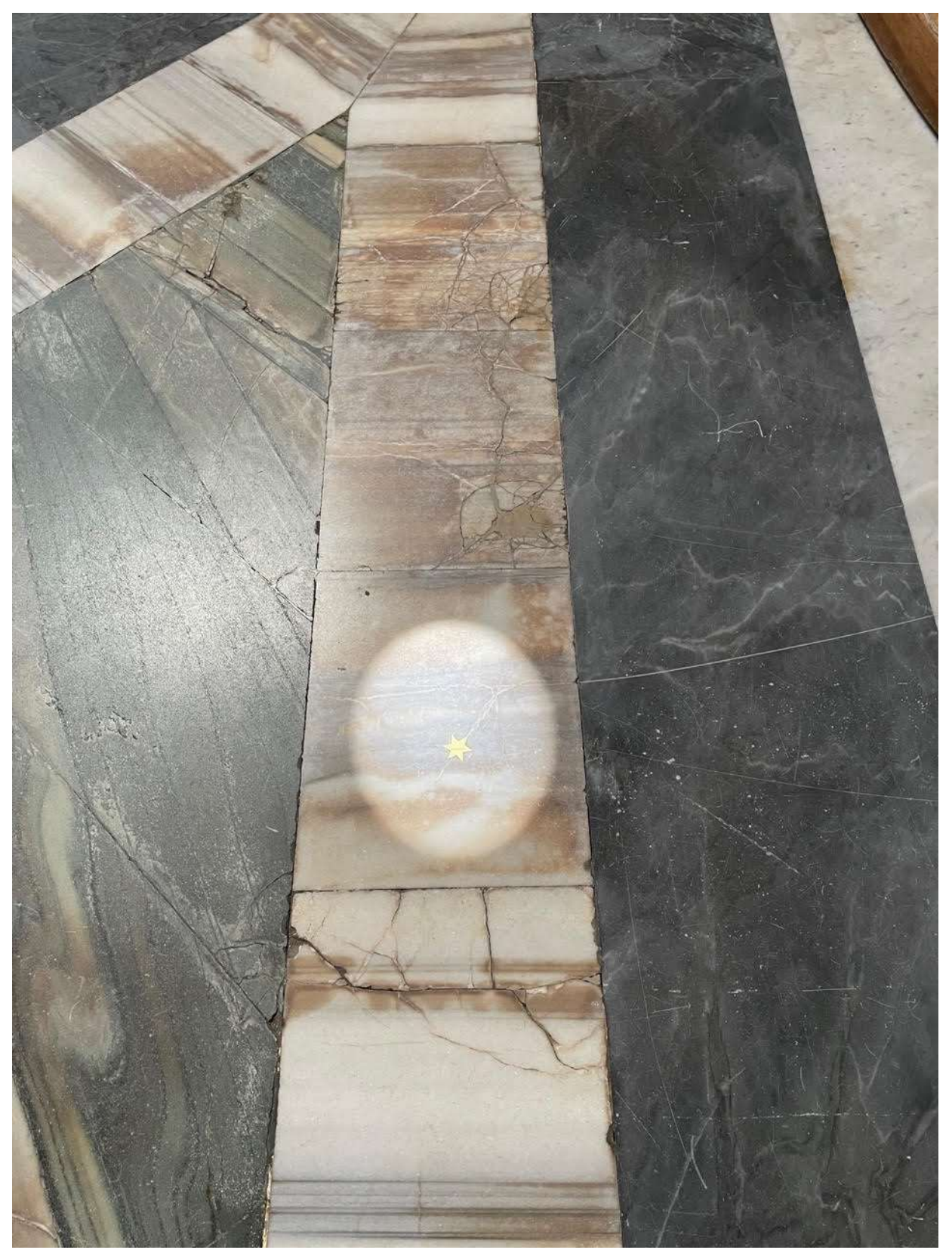

Figure 7. The first star of the papal sequence to celebrate the visit of 20 August 1702 of Clement XI, photo of Heike Baranowsky of the solar image on it on 23 April 2021 h. 11:23:31

The star was centered by the solar image exactly $3.25 \mathrm{~s}$ before the photo time, i.e. at 11:23:28. This is the result of the proportion between 220 pixel of lateral diameter of the solar image and 5 pixel of the star's shift with respect to the center of the solar image, and $143 \mathrm{~s}$ of solar transit time measured in these days. The solar meridian transit of 23 April occurred on the meridian line at 13:08:28, precisely $1 \mathrm{~h} 45 \mathrm{~m} 00 \mathrm{~s}$ after the passage of its center on that star. This star of the papal sequence is therefore placed on the floor with an astonishing precision. It tells us that the meridian line at the moment of the papal visit of 20 August 1702 was already traced in the way we see now, with the same deviation Eastward, which delays the observed transit of about $13 \mathrm{~s}$ with respect to the true North-South line, and the ephemerides. 
The difference in $4.4 \mathrm{~mm}$ Southward in the position of the center of the present pinhole with respect to the 1702 one corresponds to $1.14 \mathrm{~mm}$ left of the solar image in figure 7 with respect to what happened in 1702 and $4.25 \mathrm{~mm}$ upward. The upward shift does not change the azimuth of the Sun, while the $1.14 \mathrm{~mm}$ changes the timing of $+0.7 \mathrm{~s}$. The conclusions on the precision alignment of that papal star remain valid up to $1 \mathrm{~s}$ over $6300 \mathrm{~s}(1 \mathrm{~h} 45 \mathrm{~m})$.

\section{Conclusions and perspectives}

This study on the meridian line of s. Maria degli Angeli, the Clementine Gnomon, made by Francesco Bianchini in 1701-1702 and dedicated to the pope Clement XI (1700-1721), has been based upon real observations of the Sun, nearly 200 observations made along 900 days.

The marks on the meridian line have been measured with a total station, and controlled again with a steel meter and with a laser meter. Fluctuations of a few millimiters have been found, and evidenced when compared with astrometric measurements of the Sun.

The table of the reference marks published in table 1 has been used for data reduction. There are still some differences between observations and ephemerides as it has been verified for marks 55 , with respect to 60 , which contributed to the differences between the calculated ingress times into the zodiacal signs and the ephemerides, published in table 2.

IGEA campaign started in October 2018, and the last measures were done in solitude, with the Basilica closed to the public.

According to this calibration the center of the present pinhole is shifted $4.4 \pm 0.1 \mathrm{~mm}$ South with respect to the 1702 one.

The first star of the 20 August 1702 sequence on the floor of the Basilica shows a precision on its alignment to the nearest second, to point the $1 \mathrm{~h} 45 \mathrm{~m}$ instant to the solar meridian transit.

The large database acquired, at the moment, can be used only in clusters, i.e. to compare measurements referred to the same mark, obtained in the same way (i.e. from the end or from the beginning of the line, with the steel meter or the total station). This use shows clearly that the measurements taken at the meridian line are precise as a single millimiter or less. The corresponding difference with the ephemerides in arcsecs are well within \pm 4 " also in summer time, when the solar image is five times smaller than in winter, where the accuracy is within \pm 1 " and the imaging capability of the pinhole allows to see the larger sunspots.

After the general check of our data, we will associate both the distances of the two centers of the pinholes (the 1702 original pinhole, and the present one) to the most reliable permanent marks on the meridian line.

Finally the database of the hundreds observations made in the IGEA campaign will be translated and published with respect to the aforementioned permanent marks, chosen to be at least $5 \mathrm{~m}$ from each other.

\section{References}

1. F. Bianchini, De Nummo et Gnomone Clementino, Romae (1703)

2. C. Sigismondi, Lo Gnomone Clementino, Gerbertus 7, 1-78 (2014)

3. M. Catamo and C. Lucarini, Il Cielo in Basilica, Arpa Agami, Roma (2012)

4. J. L. Heilbron, The Sun in the Church, Harvard University Press (1999)

5. C. Sigismondi, Reference system effects detected with meridian astronomy on the Clementine Gnomon (Rome, 1702), ECU 2021 Conference Proceeding Papers (2021) 
Acknowledgments This work is especially dedicated to celebrate the 50 years of the presbyteral ordination of don Franco Cutrone (April, 18 2021), pastor of the Basilica of Santa Maria degli Angeli e dei Martiri in Rome.

Special thanks to Laura Lamberti, Letizia Bartolomeo, Giorgio Rossi, don Paolo Bettonagli, Elisa Cicillini, Claudia Caldarella, Sara Bacchini, Beatrice Baschetti, Massimiliano Romanello and Luigi Bordoni for their studies which paralleled this work. The students of 1CT and 1DT 2018/19 of Galileo Ferraris Institute, for two school years, followed fruitfully this campaign as curricular lectures of the Physics Laboratory. The contribution of all was to set up new techniques of observations and analysis. I want also to remember Prof. Carmen Gonzalez who taught mathematics at the Cervantes Institute of Rome. She sustained with enthusiasm the study of gnomonics with the students "on field" at the Basilica, and she died untimely in 2020 when based in Paris. 